\title{
A possible role for sarcosine in the management of schizophrenia
}

\author{
David Curtis
}

\section{Summary}

Sarcosine, which is freely sold as a dietary supplement, has pharmacological activity to boost functioning of the glutamatergic $\mathrm{N}$-methyl-D-aspartate receptor (NMDAR) and hence it is a biologically rational treatment for schizophrenia. The small number of studies carried out to date provide some evidence for its efficacy and psychiatrists could consider suggesting its use to their patients.

\section{Declaration of interest}

D.C. is involved in schizophrenia-related genetics research that implicates the same system as is targeted by sarcosine.

\section{Keywords}

Sarcosine; schizophrenia; glutamate; glycine; pharmacology.

\section{Copyright and usage}

(C) The Royal College of Psychiatrists 2019.
David Curtis is an Honorary Professor in the Centre for Psychiatry at Queen Mary University of London and in the UCL Genetics Institute. He is a retired Consultant Psychiatrist who carries out research in statistical and psychiatric genetics.

As summarised recently, ${ }^{1,2}$ there is convergent and compelling evidence from pharmacological, autoimmune and genetic studies that impaired functioning of the glutamatergic $N$-methyl-D-aspartate receptor (NMDAR) can produce psychotic symptoms and is sometimes involved in the pathogenesis of schizophrenia. As well as binding glutamate, NMDAR possesses a modulatory site at which glycine acts as a co-agonist. Thus, this site represents a rational therapeutic target for the treatment of schizophrenia.

\section{Bitopertin and sarcosine as GlyT-1 inhibitors}

One method whereby NMDAR activity could be enhanced is through increasing the availability of synaptic glycine by the attenuation of glycine reuptake through glycine transporter 1 (GlyT-1). Bitopertin is a glycine reuptake inhibitor and in the recent CandleLyte phase II/III trial it was compared with placebo and olanzapine as monotherapy for individuals with an exacerbation of schizophrenia. ${ }^{3}$ This study failed to achieve separation from placebo for the main outcome measures by either olanzapine or bitopertin, reflecting that participants in all three groups improved following admission to hospital. However, it was noted that more participants in both the bitopertin $30 \mathrm{mg}$ group (51\%) and the olanzapine $15 \mathrm{mg}$ group (53\%) than in the placebo group (33\%) were ready for hospital discharge at week 4 . Bitopertin was safe and well tolerated.

Another inhibitor of GlyT-1 is $N$-methylglycine (sarcosine). ${ }^{4}$ This has been the subject of a number of small trials in the treatment of schizophrenia which are detailed in the supplementary material (available at https://doi.org/10.1192/bjp.2019.194). The standard dose used is $2 \mathrm{~g} /$ day, although it is sometimes suggested that this is worked up to gradually. In some studies sarcosine on its own or as adjunctive therapy is superior to placebo and in others there are no significant differences. In particular, there is some suggestion that sarcosine may produce improvement in negative as well as positive symptoms. The most recent and largest (although still with only 30 patients per arm) is the PULSAR study, and this also had a longer follow-up period than the others, of 6 months. ${ }^{5}$ In this study, individuals with paranoid schizophrenia treated with additional sarcosine as compared with placebo had improved outcomes and higher a response rate. In the PULSAR study, 2 out of 30 participants developed hypomania following addition of sarcosine to their usual treatment. The first was also receiving quetiapine $500 \mathrm{mg}$ and citalopram $10 \mathrm{mg}$ and the second was also receiving olanzapine $25 \mathrm{mg}$ and venlafaxine $75 \mathrm{mg}$. Both episodes resolved satisfactorily following adjustment of dosage, consisting of reducing the sarcosine to $1 \mathrm{~g}$ in the former and the dose of venlafaxine to $37.5 \mathrm{mg}$ in the latter. Aside from these two individuals, all studies consistently report side-effects as being mild, transient and not clearly related to treatment.

\section{Availability of sarcosine and discussions with patients}

Sarcosine occurs naturally in a range of foods and is sold without restriction. For example, $100 \mathrm{~g}$ at $98 \%$ purity can be purchased online for around $£ 20$ (US\$26). It is widely promoted on the internet as a 'brain health' supplement (e.g. https://brainvitaminz.com) for a variety of mental health problems (https://selfhacked.com/blog/ sarcosine/, https://www.reddit.com/r/Anhedonia_Recovery/comments/77vvyz/sarcosine_nac_nacetylcysteine_success_stories_for/) and specifically for schizophrenia (http://www.schizophrenia.com/ glycinetreat.htm).

Sarcosine differs from the drugs that can be prescribed to treat schizophrenia in that patients can obtain it for themselves. Even though it is sold as a dietary supplement, there is reasonable evidence that it has a real pharmacological effect that may produce useful benefits in some patients. Psychiatrists should know how they would respond if a patient asks whether they should try taking sarcosine in addition to their antipsychotic medication. They could even consider whether they should actively recommend it. In fact, since it does not require a prescription, any member of the multidisciplinary team might have this role and mental health services may well want to develop agreed policies on communicating what they regard as the benefits and risks. It should be borne in mind that some patients may see sarcosine as a relatively attractive option. They may regard it as a 'natural' product and they may feel more autonomy in consuming something that they purchase for themselves rather than only taking a medication that is prescribed to them. Both doctors and patients may be attracted to the idea that sarcosine is a rational treatment, given that it seems to act by enhancing the functioning of a receptor that is impaired in schizophrenia. Including sarcosine as part of the treatment package may 
be seen as implementing a more holistic approach than simply prescribing antipsychotic medication on its own. It might even to some extent be perceived as a lifestyle intervention and as a part of a 'healthy diet'.

\section{Recommendations for research and practice}

It seems clear that there is a need for larger trials to produce a better understanding of the likely benefits and risks of sarcosine treatment. In terms of current practice, it seems that there is reasonable evidence that it can produce an improvement in schizophrenia symptoms when added to conventional antipsychotic treatment. Indeed, the evidence in favour of sarcosine is arguably already stronger than for some of the other interventions offered by mental health services. It seems to be almost universally well tolerated with an absence of significant side-effects, with the exception of two cases of transient hypomania in patients who were taking antidepressants. These cases suggest that caution should be exercised in patients taking serotonergic medication. A potential risk is that patients might try taking sarcosine instead of, rather than as well as, their usual medication. This could well lead to deterioration or relapse and patients should be advised against trying this without close supervision. Another risk is that patients with unsatisfactory symptom control might try to self-medicate with high doses. It is unclear what, if any, problems this might cause but it seems sensible to advise caution. A final suggestion is that in discussions with patients, carers and other health professionals one should always speak of sarcosine as enhancing the activity of glutamate receptors rather than NMDA receptors, since otherwise there will inevitably be people who gain the impression that one is referring to receptors for ecstasy.

Individual professionals and services will draw their own conclusions but it seems reasonable to conclude that suggesting sarcosine to patients with schizophrenia would be a defensible, evidence-based intervention.
David Curtis (D), MA, MD, PhD, FRCPsych, Honorary Professor, Centre for Psychiatry, Queen Mary University of London; and Honorary Professor, UCL Genetics Institute, Darwin Building, Gower Street, London WC1E 6BT, UK. Email: d.curtis@ucl.ac.uk

First received 31 Jan 2019, final revision 17 Jul 2019, accepted 5 Aug 2019

\section{Acknowledgement}

Thanks to James Innes for helpful comments.

\section{Supplementary material}

Supplementary material is available online at https://doi.org/10.1192/bjp.2019.194.

\section{References}

1 Balu DT. The NMDA receptor and schizophrenia: from pathophysiology to treatment. Adv Pharmacol 2016; 76: 351-82.

2 Tsavou A, Curtis D. In-silico investigation of coding variants potentially affecting the functioning of the glutamatergic $\mathrm{N}$-methyl-D-aspartate receptor in schizophrenia. Psychiatr Genet 2019: 29: 44-50.

3 Bugarski-Kirola D, Wang A, Abi-Saab D, et al. A phase II/III trial of bitopertin monotherapy compared with placebo in patients with an acute exacerbation of schizophrenia - Results from the CandleLyte study. Eur Neuropsychopharmacol 2014; 24: 1024-36.

4 Herdon HJ, Godfrey FM, Brown AM, et al. Pharmacological assessment of the role of the glycine transporter GlyT-1 in mediating high-affinity glycine uptake by rat cerebral cortex and cerebellum synaptosomes. Neuropharmacology 2001; 41: 88-96.

5 Strzelecki D, Urban-Kowalczyk M, Wysokiński A. Serum levels of TNF-alpha in patients with chronic schizophrenia during treatment augmentation with sarcosine (results of the PULSAR study). PSychiatry Res 2018; 268: 447-53. 